\title{
ON THE WILLMORE ENERGY OF CURVES UNDER SECOND ORDER INFINITESIMAL BENDING
}

\author{
MARIJA S. NAJDANOVIĆ AND LJUBICA S. VELIMIROVIĆ
}

Received 02 November, 2016

\begin{abstract}
The change of the Willmore energy, as a special case of so-called Helfrich energy, under second order infinitesimal bending of curves in a three-dimensional Euclidean space is studied. The first and the second variation of the Willmore energy are obtained.
\end{abstract}

2010 Mathematics Subject Classification: 53A04; 53C45

Keywords: second order infinitesimal bending, first variation, second variation, Willmore energy, curve

\section{INTRODUCTION}

Infinitesimal bending of curves and surfaces is a part of the more general bending theory, which presents one of the main consisting parts of the global differential geometry. A concept of infinitesimal bending dealt first with infinitesimal bending of surfaces and then with the same problem in the theory of curves and manifolds.

Infinitesimal bending is determined by the stationarity of arc length $s$ with appropriate precision, which is described with the condition

$$
d s_{\epsilon}^{2}-d s^{2}=o\left(\epsilon^{m}\right), m \geq 1, \epsilon \geq 0, \epsilon \rightarrow 0 .
$$

Under infinitesimal bending, geometric magnitudes describing a curve and a surface are changing and this change is determined by the variation. The variation is very important in description of the flexibility and rigidity of curves and surfaces under different kinds of deformations.

The Helfrich energy is geometrical magnitude describing the curve. It is given with the equation

$$
H_{\lambda}(C)=\frac{1}{2} \int_{I}\left(k-c_{0}\right)^{2} d s+\lambda L(C)
$$

where $k$ denotes the curvature of a curve $C, s$ is the arc length and $L(C)=\int_{I} d s$ is the length of $C$. The map $c_{0}: I \mapsto \mathbb{R}, I \subseteq \mathbb{R}$ is called spontaneous curvature.

The second author was supported by the research project 174012 of the Serbian Ministry of Science. 
The constant $\lambda \in \mathbb{R}$ is taken to be positive, so that the growth in length of a curve is penalized. The above functional is motivated by the elasticity of cell membranes [5].

The special case where $c_{0}=0$ and $\lambda=0$ is known as Willmore (bending) energy

$$
W(C)=\frac{1}{2} \int_{I} k^{2} d s,
$$

and it can also be historically motivated by the so-called Euler-Bernoulli model of elastic rods [13]. The most famous model of thin elastic rods is Euler's elastica, which is the solution of a variational problem that minimizes the Willmore energy of a thin inextensible wire [11]. The elastica is also the mathematical model of the largescale configurations of deoxyribonucleic acid (DNA) molecules [12]. In [8] isometric extremals of rotation were studied which are connected with functional $\int_{I} k d s$.

The Willmore energy has been extensively investigated analytically and numerically in recent years and it is very interesting for research yet. In [3], the authors study the long-time evolution of regular open curves in $\mathbb{R}^{n}, n \geq 2$, moving according to the $L^{2}$-gradient flow for a generalization of the Helfrich functional. In [2], the authors examine the equilibrium conditions of a curve in space when a local energy penalty is associated with its extrinsic geometrical state characterized by its curvature and torsion. To do this, they tailor the theory of deformations to the Frenet-Serret frame of the curve. In [9], the change of the Willmore energy of a curve under infinitesimal bending of the first order is analyzed. Many papers are dedicated to the Willmore energy of deformed surfaces (see $[6,15,16])$. Some other quantities are also interesting for studying at various deformations. For instance, a linear deformation technique preserving the total curvature of the masterpiece is presented in [1].

This paper deals with the first and the second variation of the Willmore energy under second order infinitesimal bending of curves. It represents a natural extension of our previous paper [10] in which the second order infinitesimal bending of curves was thoroughly studied.

\section{SECOND ORDER INFINITESIMAL BENDING OF A CURVE IN $\mathbb{R}^{3}$}

Let us consider a regular curve

$$
C: \mathbf{r}=\mathbf{r}(s)=\mathbf{r}[u(s)], \quad s \in I=[0, L] \subseteq \mathbb{R},
$$

of a class $C^{\alpha}, \alpha \geq 3$, parameterized by the arc length $s$ and included in a family of the curves

$$
C_{\epsilon}: \tilde{\mathbf{r}}(s, \epsilon)=\mathbf{r}_{\epsilon}(s)=\mathbf{r}(s)+\epsilon^{(1)}(s)+\epsilon^{2(2)}(s),
$$

where $\epsilon \geq 0, \epsilon \rightarrow 0$ and we get $C$ for $\epsilon=0\left(C=C_{0}\right)$. The fields $\stackrel{(j)}{\mathbf{z}}(s) \in C^{\alpha}$, $\alpha \geq 3, j=1,2$, are continuous vector functions defined in the points of $C$. 
Definition 1 ([4]). Family of curves $C_{\epsilon}$ is an infinitesimal bending of the second order of the curve $C$ if

$$
d s_{\epsilon}^{2}-d s^{2}=o\left(\epsilon^{2}\right) .
$$

The field $\stackrel{(j)}{\mathbf{Z}}=\stackrel{(j)}{\mathbf{Z}}(s)$ is the infinitesimal bending field of the order $j, j=1,2$ of the curve $C$.

The previous condition is equivalent to the system of equations ([4]):

$$
d \mathbf{r} \cdot d^{(1)} \mathbf{z}^{\prime}=0, \quad 2 d \mathbf{r} \cdot d^{(2)} \mathbf{z}+d^{(1)} \mathbf{z} \cdot d^{(1)} \mathbf{z}=0,
$$

where $\cdot$ stands for the scalar product in $\mathbb{R}^{3}$. This system is equivalent to the next system of differential equations:

$$
\mathbf{r}^{\prime} \cdot \mathbf{z}^{(1)}{ }^{\prime}=0, \quad 2 \mathbf{r}^{\prime} \cdot{ }^{(2)} \mathbf{z}^{\prime}+\stackrel{(1)}{(1)} \cdot \stackrel{(1)}{\mathbf{z}}^{\prime}=0,
$$

where prime denotes a derivative with respect to arc length $s$.

Let $\left\{\mathbf{t}, \mathbf{n}_{\mathbf{1}}, \mathbf{n}_{\mathbf{2}}\right\}$ be an orthonormal basis along the curve $C$, where $\mathbf{t}$ is the unit tangent, $\mathbf{n}_{1}$ and $\mathbf{n}_{2}$ are unit principal normal and binormal vector field of the curve, respectively. We choose an orientation with $\mathbf{n}_{2}=\mathbf{t} \times \mathbf{n}_{1}$.

As the vector fields $\stackrel{(1)}{\mathbf{z}}$ and $\stackrel{(2)}{\mathbf{z}}$ are defined in the points of the curve $C$, they can be presented in the form

$$
\stackrel{(j)}{\mathbf{Z}}=\stackrel{(j)}{z} \mathbf{t}+\stackrel{(j)}{z}{ }_{1} \mathbf{n}_{1}+\stackrel{(j)}{z}{ }_{2} \mathbf{n}_{2}, \quad j=1,2,
$$

where $\stackrel{(j)}{z}, \underset{z}{z_{1}}, \underset{z_{2}}{(j)}$ are the functions of $s$.

Theorem 1 ([10]). Necessary and sufficient conditions for the fields ${ }_{\mathbf{Z}}^{(j)}(2.4), j=$ 1,2 , to be infinitesimal bending fields of the corresponding order of a curve $C$ are

$$
\begin{aligned}
& \stackrel{(1)}{z}{ }^{\prime}-k \stackrel{(1)}{z}_{1}=0, \\
& \stackrel{(2)}{z}{ }^{\prime}-k \stackrel{(2)}{z_{1}}=-\frac{1}{2}\left\{\left[k^{(1)}{ }^{\prime}+\stackrel{(1)}{z_{1}}{ }^{\prime}-\tau z_{2}\right]^{2}+\left[\tau z_{1}^{(1)}+\stackrel{(1)}{z_{2}}\right]^{2}\right\},
\end{aligned}
$$

where $k$ is the curvature and $\tau$ is the torsion of $C$.

Under infinitesimal bending geometric magnitudes of the curve are changing which is described with variations of these geometric magnitudes.

Definition 2 ([14]). Let $A=A(s)$ be a magnitude which characterizes a geometric property on the curve $C$ and $A_{\epsilon}=A_{\epsilon}(s)$ the corresponding magnitude on the curve $C_{\epsilon}$ being infinitesimal bending of the curve $C$ and let the equation

$$
\Delta A=A_{\epsilon}-A=\epsilon \delta A+\epsilon^{2} \delta^{2} A+\ldots+\epsilon^{n} \delta^{n} A+\ldots
$$


be a valid one. The coefficients $\delta A, \delta^{2} A, \ldots, \delta^{n} A, \ldots$ are the first, the second, ..., the n-th variation of the geometric magnitude $A$, respectively under the infinitesimal bending $C_{\epsilon}$ of the curve $C$.

This method was used for infinitesimal $F$-planar transformations [7].

In this paper we will consider the first and the second variation of a magnitude under infinitesimal bending of the second order. For this reason, we can represent the magnitude $A_{\epsilon}$ by neglecting the terms in $\epsilon^{n}, n \geq 3$.

It is easy to prove that the first and the second variation of a line element $d s$ are equal to zero, i. e. $\delta(d s)=\delta^{2}(d s)=0$.

Theorem 2 ([10]). Under a second order infinitesimal bending of a curve $C$, the first and the second variation of the curvature are respectively

$$
\begin{aligned}
& \delta k=k^{\prime(1)} z+\stackrel{(1)}{z}_{1}^{\prime \prime}+\left(k^{2}-\tau^{2}\right) \stackrel{(1)}{z_{1}}-2 \tau z_{2}^{\prime}{ }^{\prime}-\tau^{\prime} z_{2}^{(1)}, \\
& \delta^{2} k=-\frac{k}{2}\left(\tau z_{1}^{(1)}+\stackrel{(1)}{z_{2}}\right)^{2}+\frac{1}{2 k}\left(k \tau \stackrel{(1)}{z}+2 \tau \stackrel{(1)}{z_{1}}{ }^{\prime}+\tau^{\prime}{ }_{z_{1}}^{(1)}+\stackrel{(1)}{z_{2}}{ }^{\prime \prime}-\tau^{2}{ }_{z_{2}}^{(1)}\right)^{2} \\
& +\left(k^{(2)} z+\stackrel{(2)}{z} z_{1}^{\prime}-\tau z_{2}\right)^{\prime}-\tau\left({ }^{(2)} z_{2}^{\prime}+\tau(2),\right.
\end{aligned}
$$

where $k$ is the curvature and $\tau$ is the torsion of $C$.

\section{THE WILLMORE ENERGY UNDER INFINITESIMAL BENDING}

Let us consider the Willmore energy of the curve $C(2.1)$ :

$$
W=\frac{1}{2} \int_{I} k^{2} d s
$$

The Willmore energy of deformed curve is

$$
W_{\epsilon}=\frac{1}{2} \int_{I} k_{\epsilon}^{2} d s_{\epsilon}=\frac{1}{2} \int_{I}\left(k+\epsilon \delta k+\epsilon^{2} \delta^{2} k\right)^{2}\left(d s+\epsilon \delta d s+\epsilon^{2} \delta^{2} d s\right) .
$$

As it is $\delta d s=\delta^{2} d s=0$, we have

$$
W_{\epsilon}=W+\epsilon \int_{I} k \delta k d s+\epsilon^{2} \int_{I}\left(\frac{1}{2}(\delta k)^{2}+k \delta^{2} k\right) d s,
$$

which is obtained after neglecting the terms in $\epsilon^{3}$ and $\epsilon^{4}$. Therefore,

$$
\delta W=\int_{I} k \delta k d s, \quad \delta^{2} W=\int_{I}\left(\frac{1}{2}(\delta k)^{2}+k \delta^{2} k\right) d s .
$$

Theorem 3. Under a second order infinitesimal bending of a curve $C$, the first variation of the Willmore energy is

$$
\begin{aligned}
\delta W= & \int_{I}\left[\left(k^{\prime \prime}+\frac{1}{2} k^{3}-k \tau^{2}\right) \stackrel{(1)}{z_{1}}+\left(2 k^{\prime} \tau+k \tau^{\prime}\right) z_{2}^{(1)}\right] d s \\
& +\int_{I}\left[\frac{1}{2} k^{2}{ }^{(1)} z-k^{\prime}{ }^{(1)}{ }_{1}+k z_{1}{ }^{\prime}-2 k \tau z_{2}\right]^{\prime} d s .
\end{aligned}
$$


Proof. Applying the results (2.7) and (3.2) we get

$$
\delta W=\int_{I} k\left[k^{\prime}{ }_{z}^{(1)}+\stackrel{(1)}{z_{1}}{ }^{\prime \prime}+\left(k^{2}-\tau^{2}\right) z_{1}^{(1)}-2 \tau \stackrel{(1)}{z_{2}}-\tau^{\prime}{ }_{2}^{(1)}\right] d s .
$$

Obviously, the equation

$$
k z_{1}^{\prime \prime}=\left(k z_{1}^{\prime}\right)^{\prime}-k^{\prime} z_{1}^{\prime},
$$

is valid and also

$$
-k^{\prime(1)} z_{1}^{\prime}=k^{\prime \prime} z_{1}^{(1)}-\left(k^{\prime} z_{1}^{(1)}\right)^{\prime}
$$

Further,

$$
-2 \tau z_{2}^{(1)} k=\left(-2 \tau z_{2} k\right)^{\prime}+(2 \tau k)^{\prime} z_{2}^{(1)} .
$$

Also, using the first equation in (2.5), we obtain

$$
k k^{\prime(1)} z=\left(\frac{1}{2} k^{2} \underset{z}{(1)}\right)^{\prime}-\frac{1}{2} k^{2} \underset{z}{(1)}{ }^{\prime}=\left(\frac{1}{2} k^{2} \underset{z}{(1)}\right)^{\prime}-\frac{1}{2} k^{3} z_{1}^{(1)} .
$$

Substituting the results (3.5)-(3.8) into the equation (3.4) we confirm the validity of the equation (3.3).

Directly from the previous theorem, in the case of the plane curve $(\tau=0)$, we obtain the next corollary.

Corollary 1. Under a second order infinitesimal bending of a plane curve, the first variation of the Willmore energy is

$$
\delta W=\int_{I}\left(k^{\prime \prime}+\frac{1}{2} k^{3}\right) z_{1}^{(1)} d s+\int_{I}\left[\frac{1}{2} k^{2}{ }_{z}^{(1)}-k^{\prime} z_{1}^{(1)}+k z_{1}^{\prime}\right]^{\prime} d s .
$$

From equation (3.3) we can see that the second summand is an integral of a total derivative. Also, we obtain the Euler-Lagrange equations

$$
\begin{aligned}
k^{\prime \prime}+\frac{1}{2} k^{3}-k \tau^{2} & =0, \\
2 k^{\prime} \tau+k \tau^{\prime} & =0 .
\end{aligned}
$$

These equations are the governing ones of the curve that minimize the integral (3.3). After integrating equation (3.10), we obtain

$$
\tau k^{2}=\text { const }
$$

which determines $\tau$ as a function of $k$. If we substitute $\tau$ into the equation (3.9) we get a second order differential equation for $k$ which is, obviously, integrable.

Theorem 4. Under a second order infinitesimal bending of a curve $C$, the second variation of the Willmore energy is

$$
\delta^{2} W=I_{1}+I_{2},
$$


where

$$
\begin{aligned}
& I_{1}=\int_{I} \frac{1}{2}\left[k^{\prime} \stackrel{(1)}{z}+\stackrel{(1)}{z_{1}}{ }^{\prime \prime}+\left(k^{2}-\tau^{2}\right) \stackrel{(1)}{z_{1}}-2 \tau{ }_{z_{2}}^{\prime}-\tau^{\prime} z_{2}\right]^{2} \\
& -\frac{3}{4}\left(k \tau \stackrel{(1)}{z_{1}}+k \stackrel{(1)}{z_{2}}\right)^{2}+\frac{1}{2}\left(k \tau \stackrel{(1)}{z}+2 \tau \stackrel{(1)}{z_{1}}{ }^{\prime}+\tau^{\prime} \stackrel{(1)}{z_{1}}+\stackrel{(1)}{z_{2}}{ }^{\prime \prime}-\tau^{2} z_{2}^{(1)}\right)^{2} \\
& -\frac{1}{4}\left(k^{2} \stackrel{(1)}{z}+k z_{1}^{\prime}-k \tau z_{2}\right)^{2} d s, \\
& I_{2}=\int_{I}\left[\left(k^{\prime \prime}+\frac{1}{2} k^{3}-k \tau^{2}\right) z_{1}^{(2)}+\left(2 k^{\prime} \tau+k \tau^{\prime}\right) z_{2}^{(2)}\right] d s \\
& +\int_{I}\left[\frac{1}{2} k^{2} \stackrel{(2)}{z}-k^{\prime} z_{1}^{(2)}+k \stackrel{(2)}{z_{1}}-2 k \tau z_{2}^{(2)}\right]^{\prime} d s
\end{aligned}
$$

Proof. Based on the equations (2.7), (2.8) and (3.2) we get

$$
\begin{aligned}
& \delta^{2} W=\int_{I}\left\{\frac{1}{2}\left[k^{\prime}{ }^{(1)}{ }^{\prime}+\stackrel{(1)}{z}_{1}^{\prime \prime}+\left(k^{2}-\tau^{2}\right) \stackrel{(1)}{z_{1}}-2 \tau \stackrel{(1)}{z_{2}}{ }^{\prime}-\tau^{\prime} z_{2}^{(1)}\right]^{2}\right. \\
& +k\left[-\frac{k}{2}\left(\tau \stackrel{(1)}{z_{1}}+\stackrel{(1)}{z_{2}}\right)^{2}+\frac{1}{2 k}\left(k \tau \stackrel{(1)}{z}+2 \tau \stackrel{(1)}{z_{1}}{ }^{\prime}+\tau^{\prime} z_{1}^{(1)}+{\stackrel{(1)}{z}{ }_{2}^{\prime \prime}}^{\prime \prime} \tau^{2} z_{2}^{(1)}\right)^{2}\right. \\
& \left.\left.+\left(k^{(2)}+\stackrel{(2)}{z} z_{1}^{\prime}-\tau z_{2}\right)^{\prime}-\tau\left({ }_{z 2}^{(2)} z_{2}+\tau z_{1}^{(2)}\right)\right]\right\} d s .
\end{aligned}
$$

We can express the previous equation as $\delta^{2} W=I_{1}+I_{2}$ where

$$
\begin{aligned}
& I_{1}=\int_{I} \frac{1}{2}\left\{\left[k^{\prime} z_{2}^{(1)}+z_{1}^{(1)}{ }^{\prime \prime}+\left(k^{2}-\tau^{2}\right) z_{1}^{(1)}-2 \tau z_{2}^{(1)}{ }^{\prime}-\tau^{\prime} z_{2}^{(1)}\right]^{2}\right. \\
& \left.-\left(k \tau z_{1}^{(1)}+k z_{2}^{(1)}\right)^{2}+\left(k \tau \tau^{(1)}+2 \tau z_{1}^{\prime}{ }^{\prime}+\tau^{\prime} z_{1}^{(1)}+{ }_{z_{2}}^{(1)}{ }^{\prime \prime}-\tau^{2} z_{2}\right)^{2}\right\} d s, \\
& I_{2}=\int_{I}\left[k\left(k \stackrel{(2)}{z}+\stackrel{(2)}{z_{1}}{ }^{\prime}-\tau z_{2}^{(2)}\right)^{\prime}-k \tau\left({ }_{z_{2}}^{(2)}+\tau \stackrel{(2)}{z_{1}}\right)\right] d s .
\end{aligned}
$$

As it is

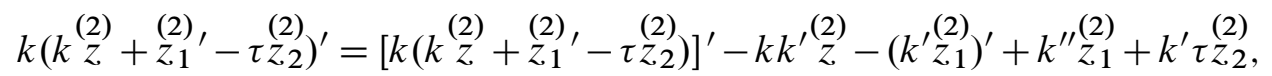

and also

$$
k \tau \stackrel{(2)}{\prime} z_{2}^{\prime}=\left(k \tau(2) z_{2}\right)^{\prime}-(k \tau)^{\prime} z_{2}^{(2)}
$$

we obtain

$$
\begin{aligned}
I_{2}= & \int_{I}\left[-k k^{\prime} \underset{z}{(2)}+\left(k^{\prime \prime}-k \tau^{2}\right) \stackrel{(2)}{z_{1}}+\left(2 k^{\prime} \tau+k \tau^{\prime}\right) z_{2}^{(2)}\right. \\
& +\int_{I}\left[k\left(k^{(2)} \underset{z}{z}+\stackrel{(2)}{z}_{1}^{\prime}-\tau z_{2}\right)-k^{\prime} z_{1}^{(2)}-k \tau z_{2}\right]^{\prime} d s .
\end{aligned}
$$

Furthermore, it holds the equation

$$
k k^{\prime(2)} z=\left(\frac{1}{2} k^{2} \stackrel{(2)}{z}\right)^{\prime}-\frac{1}{2} k^{2} \stackrel{(2)}{z},
$$


which, after using (2.5) gives

$$
k k^{\prime} \underset{z}{z}=\left(\frac{1}{2} k^{2} \stackrel{(2)}{z}\right)^{\prime}-\frac{1}{2} k^{3} \stackrel{(2)}{z}{ }_{1}+\frac{1}{4} k^{2}\left[\left(k^{(1)} z+\stackrel{(1)}{z} z_{1}^{\prime}-\tau \stackrel{(1)}{z_{2}}\right)^{2}+\left(\tau \stackrel{(1)}{z_{1}}+\stackrel{(1)}{z_{2}}\right)^{2}\right] .
$$

If we put the last summand from the previous equation into the expression for $I_{1}$, and the rest into $I_{2}$, we confirm the validity of the equations (3.12), (3.13) after a bit of calculation.

Corollary 2. Under a second order infinitesimal bending of a plane curve, the second variation of the Willmore energy is

$$
\delta^{2} W=I_{1}+I_{2}
$$

where

$$
\begin{aligned}
& \left.I_{1}=\int_{I} \frac{1}{2}\left[k^{\prime} \stackrel{(1)}{z}+\stackrel{(1)}{z_{1}}{ }^{\prime \prime}+k^{2} \stackrel{(1)}{z_{1}}\right]^{2}-\frac{3}{4}\left(k \stackrel{(1)}{z_{2}}\right)^{2}+\frac{1}{2}\left({ }_{z}^{(1)}{ }_{2}^{\prime \prime}\right)^{2}-\frac{1}{4}\left(k^{2} \stackrel{(1)}{z}+k{ }_{z}^{(1)}\right)_{1}^{\prime}\right)^{2} d s, \\
& I_{2}=\int_{I}\left(k^{\prime \prime}+\frac{1}{2} k^{3}\right) z_{1}^{(2)} d s+\int_{I}\left[\frac{1}{2} k^{2} \stackrel{(2)}{z}-k^{\prime} \stackrel{(2)}{z}_{1}+k z_{1}^{(2)}\right]^{\prime} d s .
\end{aligned}
$$

If we compare the expressions of the first variation and the part $I_{2}$ of the second variation of the Willmore energy, we conclude the Euler-Lagrange equations

$$
\begin{aligned}
k^{\prime \prime}+\frac{1}{2} k^{3}-k \tau^{2} & =0, \\
2 k^{\prime} \tau+k \tau^{\prime} & =0
\end{aligned}
$$

are the same in both cases. This means that the same governing equations of the curve minimize the integrals $\delta W$ and $I_{2}$.

It is sometimes useful to get a family of closed curves under infinitesimal bending of a closed curve (infinitesimal bending without "tearing"). In that case we must specify the condition $\mathbf{z}(0)=\mathbf{z}(L)$ for the infinitesimal bending field. Also, we suppose that the curve, as well as the infinitesimal bending field are sufficiently smooth. Keeping this in mind we have the next corollary.

Corollary 3. Under a second order infinitesimal bending of a closed curve $C$, the first and the second variation of the Willmore energy are:

$$
\begin{aligned}
& \delta W=\int_{I}\left[\left(k^{\prime \prime}+\frac{1}{2} k^{3}-k \tau^{2}\right) z_{1}^{(1)}+\left(2 k^{\prime} \tau+k \tau^{\prime}\right) z_{2}^{(1)}\right], d s \\
& \delta^{2} W=\int_{I} \frac{1}{2}\left[k^{\prime}{ }^{(1)}+\stackrel{(1)}{z_{1}}{ }^{\prime \prime}+\left(k^{2}-\tau^{2}\right) \stackrel{(1)}{z_{1}}-2 \tau \stackrel{(1)}{z_{2}}{ }^{\prime}-\tau^{\prime} z_{2}\right]^{2} \\
& -\frac{3}{4}\left(k \tau \stackrel{(1)}{z_{1}}+k \stackrel{(1)}{z_{2}{ }^{\prime}}\right)^{2}+\frac{1}{2}\left(k \tau \stackrel{(1)}{z}+2 \tau{ }^{(1)} z_{1}^{\prime}+\tau^{\prime} z_{1}+{ }_{z_{2}}^{\prime \prime}-\tau^{2} z_{2}\right)^{2} \\
& \left.-\frac{1}{4}\left(k^{2} \stackrel{(1)}{z}+k z_{1}^{\prime}-k \tau \stackrel{(1)}{z_{2}}\right)^{2}+\left(k^{\prime \prime}+\frac{1}{2} k^{3}-k \tau^{2}\right) \stackrel{(2)}{z_{1}}+\left(2 k^{\prime} \tau+k \tau^{\prime}\right) \stackrel{(2)}{z_{2}}\right] d s .
\end{aligned}
$$




\section{REFERENCES}

[1] A. Berres, H. Hagen, and S. Hahmann, "Deformations preserving Gauss curvature." in Topological and statistical methods for complex data. Tackling large-scale, high-dimensional, and multivariate data spaces. Selected papers based on the presentations at the workshop on the analysis of largescale, high-dimensional, and multivariate data using topology and statistics, Le Barp, France, June 12-14, 2013. Berlin: Springer, 2015, pp. 143-163, doi: 10.1007/978-3-662-44900-4-9.

[2] R. Capovilla, C. Chryssomalakos, and J. Guven, "Hamiltonians for curves." arXiv:nlin/0204049 [nlin.SI], p. 17, 2002, doi: 10.1088/0305-4470/35/31/304.

[3] A. Dall'Acqua and Paola Pozzi, "A Willmore-Helfrich $L^{2}$-flow of curves with natural boundary conditions." arXiv:1211.0949 [math.AP], p. 47, 2013.

[4] N. V. Efimov, "Kachestvennye voprosy teorii deformacii poverhnostei." $U M N$., vol. 3, no. 2, pp. 47-158, 1948.

[5] W. Helfrich, "Elastic properties of lipid bilayers: theory and possible experiments." $Z$. Naturforsch., vol. 28, no. c, pp. 693-703, 1973, doi: 10.1515/znc-1973-11-1209.

[6] P. Hornung, "The Willmore functional on isometric immersions." http : //www.mis.mpg.de/preprints/2012/preprint $20122_{3} 0$. pdf., p. 32, 2012.

[7] J. Mikeš, I. Hinterleitner, and J. Stranska, "Infinitesimal $F$-planar transformations." Russian Math. (Iz. VUZ)., vol. 52, no. 4, pp. 13-18, 2008, doi: 10.3103/s1066369x08040026.

[8] J. Mikeš, M. Sochor, and E. Stepanova, "On the existence of isoperimetric extremals of rotation and the fundamental equations of rotary diffeomorphisms." Filomat., vol. 29, no. 3, pp. 517-523, 2015, doi: 10.2298/fil1503517m.

[9] M. S. Najdanović, "Infinitesimal bending influence on the Willmore energy of curves," Filomat., vol. 29, no. 10, pp. 2411-2419, 2015, doi: 10.2298/FIL1510411N.

[10] M. S. Najdanović and L. S. Velimirović, "Second order infinitesimal bending of curves," Filomatin press.

[11] D. A. Singer, "Lectures on Elastic Curves and Rods." Aip Conference proceedings., vol. 1002, no. 3, pp. 1-30, 2008, doi: 10.1063/1.2918095.

[12] E. Starostin, "Equilibrium configurations of a thin elastic rod with self-contacts." PAMM, Proc. Appl. Math. Mech., vol. 1, no. 1, pp. 137-138, 2002, doi: 10.1002/16177061(200203)1:1;137::AID-PAMM137;3.0.CO;2-B.

[13] C. Truesdell, "The influence of elasticity on analysis: The classic heritage." Bull. Am. Math. Soc., New Ser., vol. 9, pp. 293-310, 1983, doi: 10.1090/S0273-0979-1983-15187-X.

[14] I. N. Vekua, Obobschennye analiticheskie funkcii. Moskva: Nauka, 1959.

[15] L. S. Velimirović, M. S. Ćirić, and M. D. Cvetković, "Change of the Willmore energy under infinitesimal bending of membranes." Comput. Math. Appl., vol. 59, no. 12, pp. 3679-3686, 2010, doi: 10.1016/j.camwa.2010.03.069.

[16] L. S. Velimirović, M. S. Ćirić, and N. M. Velimirović, "On the Willmore energy of shells under infinitesimal deformations." Comput. Math. Appl., vol. 61, no. 11, pp. 3181-3190, 2011, doi: 10.1016/j.camwa.2011.03.035.

\section{Authors' addresses}

\section{Marija S. Najdanović}

University of Niš, Faculty of Sciences and Mathematics, 33 Višegradska, 18000 Niš, Serbia Current address: Preschool Teacher Training College, 22-24 Ćirila i Metodija, 37000 Kruševac, Serbia

E-mail address: mari jamath@yahoo.com 
Ljubica S. Velimirović

University of Niš, Faculty of Sciences and Mathematics, 33 Višegradska, 18000 Niš, Serbia

E-mail address: vljubica@pmf.ni.ac.rs 